\title{
ATTRIBUTES BALANCE ON THE ADOPTION OF PLATFORM BASED SOLUTIONS FOR SATELLITES
}

\author{
Otavio Luiz Bogossian
}

\author{
Instituto Nacional de Pesquisas Espaciais \\ Av. dos Astronautas 1758, CEP 12227-010, São José dos Campos - SP Brasil \\ otavio.bogossian@lit.inpe.br
}

\section{Geilson Loureiro}

Instituto Nacional de Pesquisas Espaciais

Av. dos Astronautas 1758, CEP 12227-010, São José dos Campos - SP Brasil

geilson@gsr.inpe.br

\begin{abstract}
This paper aims to propose the principles of a systems architecting method to assess and guide the conceptual design of platform based solutions for satellite space applications. The method helps the developers to assess the platform changeability to achieve future missions with a minimum impact in terms of modifications and to assess the platform comprehensiveness in terms of possible missions. Changeability attributes applicable to the platform aims to implement parameters like robustness, flexibility, agility, etc. showing the capacity of the platform to comply, fast and with minimum modifications, the future missions. Comprehensiveness aims to balance platform comprehensiveness attributes in terms of missions and the platform efficiency based on the additional mass necessary to cover space environment requirements like altitude (drag, radiation and torque), satellite pointing, etc. The more over dimensioned, the less efficient is the satellite. Conclusions are that the method promotes a great enhancement on the productivity of platform based solutions conception while increasing the quality of the conceptual phase results.
\end{abstract}

Keywords: Platform, satellites, multi-mission, changeability, product family

\section{Introduction}

The family of products concept became relevant with the transformation of the concept of mass production into mass customization aiming to comply with individual client needs (Pine, 1993). The segmentation market grid based on platform was introduced (Meyer, et al., 1993) as the way to leverage the family of product across different market niches. The attempt to map the evolution of the product family based on platform by means of extensions and upgrades (Meyer, et al., 1997) was proposed also. The family is a set of similar products obtained from a common platform, given to each product the functionalities required by specific clients (Meyer, et al., 1993).

The space context has specific characteristics such as the complexity of the products and the very low production volume. It was remarked that space products are designed to comply with particular mission (Gonzalez-Zugasti, et al., 2000), being a product designed for this objective contrasting with other general application in which they are designed for market niche.

The space product referenced in this paper corresponds to the satellite, usually established as a system. It is usually divided into sub-systems, each one for a specific discipline (structure, power, communication, on board data handling, etc.). Each sub-system is composed of several equipments (Aerospatiale and Sextant, 1995; Alary, et al., 2007; Buisson, et al., 1998; Bouzat, 2000; Galeazzi, 2000; INPE, 2001). As example, the power subsystem is composed of the following equipment: battery, solar panels, regulator, DC-DC converters, etc.

The space products composed of a common platform that includes usually the same components to all space products (satellites) and a set of specific components that characterize each particular product and mission are according to what was proposed for general application product based on a platform (Meyer, et al., 1993). The platform for satellite is usually composed of all components necessary to guarantee the satellite operation (structure, thermal, power, on board data handling, attitude control, communication for control purpose, propulsion, etc.). The mission specific components include scientific experiments, cameras (Earth or Sun observation), communication for the specific application, sensors, etc. (Aerospatiale and Sextant, 1995; Alary, et al., 2007; Buisson, et al., 1998; Bouzat, 2000; Dechezelles, et al., 2000; INPE, 2001). 
This paper aims to present and justify the elements of a method for assessing space platform development while it is being developed, to guide the various decision making points during space platform design. This paper has the following specific objectives:

a) To analyze up-to-date efforts on general application and space platform development;

b) To explain the changeability basic elements of the method applicable to the space platforms;

c) To explain the comprehensiveness basic elements of the method;

d) To provide an initial idea on how these elements will be used in the method.

In order to achieve these objectives, this paper is organized as following. Section 2 presents the general application platform effort and the current status of space platform development. Section 3 presents the Changeability elements. Section 4 presents the Comprehensiveness elements. Section 5 shows how the method can be built from these elements. Section 6 draws some conclusions and sets up some further work.

\section{Development process for satellite family based on platforms}

The product family in the general context applications could be developed based on a set of modular components (Gonzalez-Zugasti, et al., 2000; Du, et al., 2001; Ulrich, 1995), referred as configurational product family design by some authors. Each product of the family is developed adding, replacing or subtracting one or more functional modules.

Another approach is applying the scalability or parametric configuration (Simpson, et al., 2001) in which the capacity or performance of each module could be increased or reduced according to the customer needs.

It was proposed a methodology for product portfolio definition maximizing the use of common components (Dahmus, et al., 2001). An approach from a marketing and sales perspective was proposed with the product family definition based on the functional features associated to customers groups (Agard, et al., 2004). Additional methods based on technical characteristics (De Lit, et al., 2003; Simpson, 2004), with the family members defined based on technology, manufacturing process, design parameters, assembly process, etc. were presented.

As it was shown, some papers define the product family based on design methods (modularity, platform based, configurational or scalable), others define based on variety generation methods to produce product variety to target markets and, finally, others based on technical aspects improving the product process, stock reduction, component reutilization promotion, etc.

In the space context the most common approach is the independent development (Gonzalez-Zugasti, et al., 2000), which means a product developed for the specific mission requirements. The number of recurring products is very limited, usually of one or two units. There are some exceptions like the GPS (24 satellites) and Galileu (27 satellites) constellations (Forest, 2004), where the satellites are placed in different orbits and phases. The satellite platform concept was adopted by some space programs to explore common aspects of the products, from one mission to another. They do not have a complete view of the satellite family to be generated. However they aim to increase the reutilization of the common part (platform) as much as possible when future missions are defined.

A very particular example (case of the Joint Strike Fighter program) of simultaneous development of a family of fighter planes, to define the common platform from the requirements of the all products (Boas, et al., 2006) was analyzed by some authors. They presented a second example based on the Boeing 777 family of products in which the initial product (the first plane of the family) is developed at the same time as the platform that will be the core of future planes. This approach is clearly the sequential development process. According to the authors, this approach has the inconvenience of making the first plane a strong reference for the platform design that could cause problems for the future planes. This inconvenience is mainly due to long development process and difficulties to define the different members of the family.

The sequential approach is often applied to the development of multi-mission satellite platforms. It is demonstrated by missions like Jason 1 using CNES PROTEUS platform (Aerospatiale and Sextant, 1995; Dechezelles, et al., 2000; Grivel, et al., 2000), Demeter mission using CNES Myriade Product Line platform (called previously Ligne de Produits Micro-satellite (Buisson, et al., 1998)) (Bouzat, 2000; Cussac, et al., 2004), SkyMed/COSMOS mission using ASI/Alenia PRIMA platform (Galeazzi, 2000).

The development approach for a satellite family based on a platform impose constraints in some mission parameters (e.g. orbits, pointing accuracy, available launchers, lifetime, mass and power limit for the payload, etc.) (Dechezelles, et al., 2000; Galeazzi, 2000; INPE, 2001; Grivel, et al., 2000). 


\section{Changeability}

It was proposed a Design for Changeability - DfC to challenge the technological and market dynamism (Fricke, et al., 2005) with a constant inclusion of new clients, as well as, the changing of products environment (cell phones services, GPS, Wi-Fi, etc.). During the design phase, the possibility to change the design should be open until as late as possible. They considered also modifying the product during the operation/utilization phases.

The defined aspects (DfC term) (Fricke, et al., 2005) that were considered applicable to the satellite platform design taking into account the sequential development process are the following:

Robustness - System ability to be insensitive to the environment changes. This aspect is applicable considering that the platform will be used in different mission with different launching, space environment and mission requirements.

Flexibility - System ability to be easily changed. This aspect is applicable taking into account that it is expected the platform, that is the core of all satellite products, to have the ability to be easily modified for different missions of the same category (i.e. LEO and excluding Solar System missions, GEO, etc.). This concept is applied in PRIMA platform (Galeazzi, 2000) considering yes/no and scalability options for the platform equipments.

Agility - System ability to be quickly changed. This aspect is applicable considering that it is expected that products (satellites) based on a platform, recover the development time spent during the first mission (platform and first product) at each developed product and it is also expected that the time spent on each satellite to be lower than an independent development to make viable the use of a platform.

The aspects considered in the Design for Changeability were proposed to be implemented by Basic and Extended Principles. Some of these principles correspond to axioms previously stated (Suh, 1990). The principles considered applicable to the satellite platform design taking into account the satellite family development approach, are the following:

Ideality/Simplicity - This principle aims to reduce the system complexity. This principle is applicable considering that all satellite projects are complex systems with a lot of interfaces, functions and components integrated in a limited room, with reduced mass and power consumption.

Independence - This principle aims to minimize the impact of change in design parameters. This principle is applicable considering that for each mission the satellite is composed of mission specific components and platform common components. It is expected that specific component and specific environment do not affect the platform components and the interfaces. This principle is adopted in the PRIMA platform design (Galeazzi, 2000) with the objective of thermally decoupling the specific components (payload module) from the platform components.

Modularity/Encapsulation - This principle aims to build a system architecture that clusters the system functions into various modules (components) while minimizing the coupling among them (loose coupling) and maximizing the cohesion within the module. This principle is applicable considering the specific and platform components need to be decoupled as much as possible.

Scalability - Ability to change the components increasing or reducing their capacity or performance $(e . g$. amount of data storage, capacity of angular momentum in the reaction wheels, etc.). This principle is applicable to increase the efficiency of the standard platform for the different mission requirements. This concept is applied in the PRIMA platform (Galeazzi, 2000).

Integrability - Characterized by the compatibility and interoperability among the interfaces (proprietary or open systems) by adopting standards that enable to change easily the interconnected components. This principle is applicable mainly for the power bus and on-board data interfaces that are necessary for almost all the components. It will facilitate the scalability implementation. This principle is implemented on the on-board data handling subsystem that interfaces with almost all components using a standard bus (Dechezelles, et al., 2000; Galeazzi, 2000; Grivel, et al., 2000).

Decentralization - This principle is characterized by the distribution of control, information, resources, architecture attributes or properties among the components. This principle is applicable mainly to the power bus and on-board data handling. 


\section{Mission comprehensiveness and platform efficiency}

In order to assess the mission Comprehensiveness it is necessary to reduce the scope of possible orbits. The considered orbits were based on the Myriade (CNES), Proteus (CNES), PRIMA (ASI/ALENIA) and PMM (INPE) multi-mission platforms (Buisson, et al., 1998; Galeazzi, 2000; INPE, 2001; Rougeron, 2000). The following limitation were established:

a) Only circular orbits (low eccentricity) with altitudes between 400 and $1500 \mathrm{~km}$;

b) Sun-synchronous orbits (SSO) with equator crossing time of 6:00 am and 10:00 am (also almost SSO);

c) Equatorial and low inclination orbits up to $25^{\circ}$;

d) Three orbit inclinations and three SSO with three different altitudes will be considered.

Four possible satellite configurations will be considered all with a parallelepiped shape. Two configurations with fixed solar panels with one or two wings and two with rotating solar panel with one or two wings. The hardware alternatives are the following: 1) up to three torque rods sizes for each axis, 2) up to three propellant reservoir sizes and 3) up to three reaction wheels sizes for each axis.

With respect to the satellite pointing, nadir will be considered for SSO orbits and nadir and Sun for other orbits. The following space environmental effects are considered:

a) Cumulated radiation - The impact in the component hardness in terms of krads for each possible orbit. Mission lifetime will be considered with respect to the maximum lifetime of the platform. An indirect impact on mass will be considered based on the additional price of the components and the cost to transport a unit of mass to the space;

b) Aerodynamic torque due to the residual atmosphere - It will affect the reaction wheels dimensioning in terms of angular momentum and the increment of required mass;

c) Drag due to the residual atmosphere - It will affect the propellant and reservoir mass to keep the orbit error lower than a specified value;

d) Use of the magnetic field to unload the reaction wheels - Dimensioning the torque rods and its increment in terms of mass;

e) Static and dynamic launcher environment - The increment in the structure (platform) mass necessary to consider several launchers and different payload masses;

f) Power required - For each orbit, pointing and satellite solar panels configuration, will be determined the amount of solar panel necessary to provide the minimum amount of power established for the platform as well as the battery necessary to overcome the eclipse periods. The increment of mass necessary to cover all the orbits will be considered for the solar panels and batteries.

The thermal dimensioning and the structure are not considered as part of the platform (Bouzat, 2000) due to the necessity to design for each specific mission (payload module for the structure), therefore it does not induce any inefficiency.

The launching window with respect to the solar flux cycle was not considered as inefficiency due to the necessity of the platform to be ready to launch at any time in the solar cycle.

\section{Elements of the method to be developed}

Considering the basic development approach, the method shall implement Changeability in such a way the platform will be designed with the capacity to be quickly adapted for future and unknown missions. Changeability will provide to the platform designer, at the initial design phase, enough information about the robustness, flexibility and agility of the platform. Based on the Changeability aspects and principles presented in this paper, the method shall implement objective parameters to assess the satellite configuration. In addition, specific space principles shall be considered (e.g. robustness shall include the space qualification process covering or not the different space and launcher environment, shall include design for the worst case of solar cycle, propellant reservoir dimensioned to cover or not orbit maintenance for the considered cases, etc.).

The method shall implement also the Comprehensiveness (in terms of missions) and the corresponding platform efficiency to provide to the platform designer, at the initial design phase, enough information to decide how much to pay in terms of efficiency, to implement the Comprehensiveness.

For Comprehensiveness implementation, the method shall implement a limited number of cases in terms of orbits, pointing and satellite configurations, considering those more applicable. It shall also consider some premises (orbit maximum error, reaction wheels and torque rods dimensioning, etc.) and the implementation of the scalability to give to the designer, an easy way to increase the platform efficiency. The method will consider 
as objective parameter to implement the Comprehensiveness the worst/least case of equipment mass, directly or indirectly.

The method also shall receive inputs from the platform designer, such as lifetime, mass, inertia, surface of the solar panels, etc. for Comprehensiveness and satellite architecture definitions and the qualification process for Changeability.

The method to be developed shall be easily implemented (spreadsheet or simple program) without the necessity to perform simulations to assess the platform. The simulations will be performed during the method design phase for all considered cases.

\section{Conclusions and further work}

Section 2 presented the effort to develop the platform concept for general application and also concluded the development approach applied on the development of space platforms. Section 3 presented the Changeability basic elements applied to the space platforms derived from the general application platform concept. Section 4 presented the basic Comprehensiveness elements developed, based on the application scope of some existing or in development satellite platforms. Section 5 presented the initial idea of the method implementation. The Sections 2 to 5 have presented and justified the elements of the method to assess space platform during the development phase. As conclusion, the main and specific objectives presented in the Section 1 were achieved by this paper.

To finalize the method development further work is necessary. With respect to Changeability, it is necessary to define objective parameters to assess the platform. These parameters will cover the platform architecture (e.g. for data handling) and the qualification plan (e.g. for robustness a bonus will be given to the qualification plan that cover a significant number of different environment scenarios). With respect to Comprehensiveness, it is necessary to perform several simulations and verify the mass impact on the platform components.

Finally it is necessary to produce two measures, one for Changeability and another for Comprehensiveness.

\section{References}

Aerospatiale and Sextant Filiere Proteus CNES. - 1995. - Aerospatiale/Sextant Brochure.

Agard B. and Kusiak A. Data-mining-based methodoly for the design of product families [Journal] // International Journal of Production Research. - 2004. - 15 : Vol. 42. - pp. 2955-2969.

Alary D. and Lambert H. The Myriade Product Line [Journal] // ACTA Astronautica. - 2007. - Vol. 61. - pp. 223-227.

Boas R. C. and Crawley E. F. Extending Platforming to the Sequential Development of System Families [Conference] // INCOSE - 16th Annual International Symposium Proceedings. - 2006.

Bouzat C. CNES Microsatellite Product Line, an approach for innovation [Conference] // Small Satellites Systems and Services, 5th International Symposium. - La Boule, France : [s.n.], 2000.

Buisson F [et al.] La Ligne the Produits Micro-satellite du CNES [Conference] // Small Satellites Systems and Services, 4th International Symposium. - Antibes-San Juan Les Pains, France : [s.n.], 1998.

Cussac T., Buisson F. and Parrot M. The Demeter Program: Mission and Satellite Description Early in Flight Results [Conference] // 55th International Astronautical Congress. - Vancouver, Canada : [s.n.], 2004. IAC-04-IAA.4.11.2.04.

Dahmus J. B., Gonzales-Zugasti J. P. and Otto K. N. Modular Product Architecture [Journal] // Design Studies. 2001. - Vol. 22. - pp. 409-424.

De Lit P. G. and Delchambre A. Integrated Design of a product family and its assembly system [Book]. Massachusetts : Kluwer Academic Publishers, 2003.

Dechezelles J-J and Huttin G. PROTEUS: A Multimission Platform for Low Earth Orbits [Journal] // Air \& Space Europe. - 2000. - 1 : Vol. 2. - pp. 77-81.

Du X., Jiao J. and Tseng M. M. Architecture of product family: Fundamentals and Methodology [Journal] // Concurrent Engineering: Research and Application. - 2001. - 4 : Vol. 9. - pp. 309-325.

Forest W. M. Interoperability of the GPS and Galileo Timescales for Positioning and Metrology [Conference] // European Frequency and Time Forum. - 2004. - Vol. 18. - pp. 468-475.

Fricke E. and Schulz A. P. Design for Changeability (DfC): Principles to enable changes in system throughout their entire lifecycle [Journal] // System Engineering. - 2005. - 4 : Vol. 8.

Galeazzi C. Prima: A new competitive smal satellite platform [Journal] // Acta Astronautica. - 2000. - 2-6 : Vol. 46. - pp. 379-388.

Gonzalez-Zugasti J. P., Otto K. N. and Baker J. D. A Method for Architecting Product Platforms [Journal]. 2000. - Vol. 12. - pp. 61-72. 
Gonzalez-Zugasti J. P., Otto K. N. and Baker J. D. A Method for Architecting Product Platforms [Journal] // Research in Engineering Design. - 2000. - Vol. 12. - pp. 61-72.

Grivel C. [et al.] Proteus: European Standard for small satellites [Conference] // Small Satellites System and Services, 5th International Symposium. - La Boule, France : [s.n.], 2000.

INPE Multimission Platform: Data Package for System Requirements Review [Journal]. - 2001. - Brazilian National Institute for Space Research (INPE) - internal document.

Meyer M. and Lehnerd A. P. The product family and the dynamics of core capability [Journal] // Sloan Magazine Review. - 1993. - pp. 29-47.

Meyer M. and Utterback J. The power of product platform - building value and cost leadship [Book]. - New York : Free Press, 1997.

Pine B. J. Mass customization: The new frontier in business competition [Article] // Harvard Business School Press. - 1993.

Rougeron M. CNES Minisatellite Missions/Les Missions Proteus [Conference] // Small Satellites Systems and Services, 5th International Symposium. - La Boule, France : [s.n.], 2000.

Simpson T. W. Product platform design and customization: Status and promise [Journal] // Artificial Intelligence for Engineering Design, Analysis and Manufacturing. - 2004. - 1 : Vol. 18. - pp. 3-20.

Simpson T. W., Maier J. R. A. and Mistree F. Product platform design and customization: Status and promise [Journal] // Research in Engineering Design. - 2001. - 1 : Vol. 13. - pp. 2-22.

Suh N. P. Principles of Design [Book]. - [s.1.] : Oxford University Press, 1990.

Ulrich K. The Role of product architecture in the manufacturing firm [Journal] // Research Policy. - 1995. - 3 : Vol. 24. - pp. 419-440. 